\title{
Article \\ Symmetry and Its Role in Oscillation of Solutions of Third-Order Differential Equations
}

\author{
M. Sathish Kumar ${ }^{1,+} \mathbb{D}$, Omar Bazighifan ${ }^{2,3,+} \mathbb{D}$, Khalifa Al-Shaqsi ${ }^{4,+}{ }^{\text {, Fongchan Wannalookkhee }}{ }^{5,+}$ (D) \\ and Kamsing Nonlaopon $5, *,+\mathbb{D}$
}

check for updates

Citation: Kumar, M.S.; Bazighifan, O.; Al-Shaqsi, K.; Wannalookkhee, F.; Nonlaopon, K. Symmetry and Its Role in Oscillation of Solutions of Third-Order Differential Equations. Symmetry 2021, 13, 1485. https:// doi.org/10.3390/sym13081485

Academic Editor: Calogero Vetro

Received: 14 July 2021

Accepted: 6 August 2021

Published: 13 August 2021

Publisher's Note: MDPI stays neutral with regard to jurisdictional claims in published maps and institutional affiliations.

Copyright: (c) 2021 by the authors. Licensee MDPI, Basel, Switzerland. This article is an open access article distributed under the terms and conditions of the Creative Commons Attribution (CC BY) license (https:/ / creativecommons.org/licenses/by/ $4.0 /)$.
1 Department of Mathematics, Paavai Engineering College (Autonomous), Namakkal 637 018, India; msksjv@gmail.com

2 Section of Mathematics, International Telematic University Uninettuno, Corso Vittorio Emanuele II, 39, 00186 Rome, Italy; o.bazighifan@gmail.com

3 Department of Mathematics, Faculty of Science, Hadhramout University, Hadhramout 50512, Yemen

4 Department of Information Technology, Nizwa College of Technology, University of Technology and Applied Science, P.O. Box 75, Nizwa 612, Oman; khalifa.alshaqsi@nct.edu.om

5 Department of Mathematics, Faculty of Science, Khon Kaen University, Khon Kaen 40002, Thailand; fongchan_wanna@kkumail.com

* Correspondence: nkamsi@kku.ac.th; Tel.: +668-6642-1582

+ These authors contributed equally to this work.

Abstract: Symmetry plays an essential role in determining the correct methods for the oscillatory properties of solutions to differential equations. This paper examines some new oscillation criteria for unbounded solutions of third-order neutral differential equations of the form $\left(r_{2}(\varsigma)\left(\left(r_{1}(\varsigma)\left(z^{\prime}(\varsigma)\right)^{\beta_{1}}\right)^{\prime}\right)^{\beta_{2}}\right)^{\prime}$ $+\sum_{i=1}^{n} q_{i}(\varsigma) x^{\beta_{3}}\left(\phi_{i}(\varsigma)\right)=0$. New oscillation results are established by using generalized Riccati substitution, an integral average technique in the case of unbounded neutral coefficients. Examples are given to prove the significance of new theorems.

Keywords: neutral differential equation; oscillation; Riccati substitution; deviating arguments

\section{Introduction}

We consider the third-order neutral differential equations with several delays:

$$
\left(r_{2}(s)\left(\left(r_{1}(\varsigma)\left(z^{\prime}(s)\right)^{\beta_{1}}\right)^{\prime}\right)^{\beta_{2}}\right)^{\prime}+\sum_{i=1}^{n} q_{i}(\varsigma) x^{\beta_{3}}\left(\phi_{i}(\varsigma)\right)=0, \quad \varsigma \geq \varsigma_{0}>0,
$$

where $z(\varsigma)=x(\varsigma)+p(\varsigma) x(\varrho(\varsigma)), \beta_{i}>0(i=1,2,3)$ is a ratio of odd integers. Considering the following conditions for (1) are satisfied:

$$
\left\{\begin{array}{l}
r_{i} \in C\left(\left[\varsigma_{0}, \infty\right),(0, \infty)\right) \text { and } \int_{\varsigma_{0}}^{\infty} r_{i}^{-1 / \beta_{i}}(s) d s=\infty, i=1,2 ; \\
q_{i} \in C\left(\left[\varsigma_{0}, \infty\right),[0, \infty)\right), \phi_{i} \in C\left(\left[\varsigma_{0}, \infty\right), \mathbb{R}\right) \text { and } \lim _{\varsigma \rightarrow \infty} \phi_{i}(\varsigma)=\infty, \text { where } i=1,2, \cdots n ; \\
\varrho \in C\left(\left[\varsigma_{0}, \infty\right), \mathbb{R}\right) \text { is strictly increasing, } \varrho(\varsigma)<\varsigma, \text { and } \lim _{\varsigma \rightarrow \infty} \varrho(\varsigma)=\infty ; \\
p \in C\left(\left[\varsigma_{0}, \infty\right), \mathbb{R}\right) \text { with } p(\varsigma) \geq 1, \text { and } p(\varsigma) \neq \equiv 1, \text { eventually. }
\end{array}\right.
$$

To formulate a solution for (1), we need a function $x:\left[\zeta_{x}, \infty\right) \rightarrow \mathbb{R}$ such that $z \in$ $C^{2}\left(\left[\zeta_{x}, \infty\right), \mathbb{R}\right), r_{1}\left(z^{\prime}\right)^{\beta_{1}} \in C^{1}\left(\left[\zeta_{x}, \infty\right), \mathbb{R}\right), r_{2}\left(\left(r_{1}\left(z^{\prime}\right)^{\beta_{1}}\right)^{\prime}\right)^{\beta_{2}} \in C^{1}\left(\left[\zeta_{x}, \infty\right), \mathbb{R}\right)$ and which satisfies Equation (1) on $\left[\varsigma_{x}, \infty\right)$. We only consider those solutions $\left.x(\varsigma)\right)$ of $(1)$ as defined on some ray $\left[\varsigma_{x}, \infty\right)$; for some $\varsigma_{x} \geq \varsigma_{0}$, which satisfies $\sup \{|x(\varsigma)|: \varsigma \geq T\}>0$ for every $T \geq \varsigma_{x}$. We start with the assumption that Equation (1) does possess a proper solution. A proper solution $x(\varsigma)$ of Equation (1) is said to be oscillatory if it has arbitrarily large zeros; it is called nonoscillatory otherwise. Equation (1) is termed oscillatory if all its solutions are oscillatory. 
The study of the oscillation of various classes of differential equations has recently received a lot of interest. Many authors have conducted various investigations. Some writers have focused their research on the oscillation of differential equations. Symmetry plays an important and fundamental role in the study of the oscillations of solutions to equations. There has been a growing interest in obtaining sufficient (as well as necessary) conditions for the oscillatory and asymptotic behaviour of solutions of the third order. We refer the reader to [1-10] for such results.

The oscillatory and asymptotic behavior of the solutions to (1) and various classes of equation forms such as

$$
\begin{gathered}
{\left[a(\varsigma)\left[x(\varsigma) \pm p(\varsigma) x(\delta(\varsigma))^{\prime \prime}\right]^{\gamma}\right]^{\prime}+q(\varsigma) x^{\gamma}(\tau(\varsigma))=0} \\
{\left[a(\varsigma)\left[b(\varsigma) z^{\prime}(\varsigma)\right]^{\prime}\right]^{\prime}+q(\varsigma) x(\tau(\varsigma))=0} \\
\left(r(\varsigma)\left((x(\varsigma)+p(\varsigma) x(\tau(\varsigma)))^{\prime \prime}\right)^{\alpha}\right)^{\prime}+\int_{a}^{b} q(\varsigma, \xi) x^{\alpha}(\phi(\varsigma, \xi)) d \xi=0, \\
{\left[a(\varsigma)\left[b(\varsigma) z^{\prime}(\varsigma)\right]^{\prime}\right]^{\prime}+\int_{a}^{b} q(\varsigma, \xi) x^{\alpha}(\phi(\varsigma, \xi)) d \xi=0} \\
\left(a_{2}(\varsigma)\left[\left(a_{1}(\varsigma) z^{\prime}(\varsigma)\right)^{\prime}\right]^{\lambda}\right)^{\prime}+\int_{c}^{d} \tilde{q}_{1}(\varsigma, \xi) y^{\lambda}(\varsigma-\xi) d \xi+\int_{c}^{d} \tilde{q}_{2}(\varsigma, \xi) y^{\lambda}(\varsigma+\xi) d \xi=0, \\
\left(a(\varsigma)\left(\left(x(\varsigma) \pm \sum_{i=1}^{n} p_{i}(\varsigma) x\left(\sigma_{i}(\varsigma)\right)\right)^{\prime \prime}\right)^{\alpha}\right)^{\prime}+\sum_{j=1}^{m} f_{j}\left(\varsigma, x\left(\tau_{j}(\varsigma)\right)\right)=0
\end{gathered}
$$

have been studied by many authors concerned with the case where $p$ is bounded, that is, the cases

$$
-1<p_{0} \leq p(\varsigma) \leq 0, \quad 0 \leq p(\varsigma) \leq p_{0} \leq 1, \quad 0 \leq p(\varsigma) \leq p_{0} \leq \infty,
$$

were considered (see $[2,11-21]$ and the references therein).

For $r_{1}=\beta_{1}=1, n=1$, B. Baculíková, J. Dzurina [2], J. Dzurina et al. [3], T. Li and Y. V. Rogovchenko [13] studied asymptotic behavior of solutions to Equation (1) assuming that $0 \leq p(\varsigma) \leq p_{0} \leq 1$. If $r_{1}=\beta_{1}=1$, the problem of the oscillation of Equation (1) in the case where $0 \leq p_{i} \leq 1$ and $-\mu \leq \sum_{i=1}^{n} p_{i}(\varsigma) \leq 0$ has also been discussed by A. A. Soliman et al. [20]. Recently, for $\beta_{1}=n=1$ and $0 \leq p(\varsigma) \leq p_{0} \leq \infty$, Equation (1), its particular cases and modifications have been studied by Y. Jiang et al. [11], T. Li et al. [4], R. Elayaraja et al. [12].

We noticed that, in the research mentioned above, the oscillatory and asymptotic behaviour of third-order neutral differential equations with several delays received relatively less attention, despite the reality that the deviating arguments cause a new challenge in establishing oscillatory and asymptotic criteria for them. In view of the above observations, in this paper, our aim is to obtain explicit sufficient conditions for the oscillation of all solutions of (1) via the generalized Riccati substitution in the case of $p(\varsigma) \geq 1$.

\section{Main Results}

We start with the following lemmas, which are required for our theorem proofs. Throughout this paper, we will be using the following notation: 


$$
\begin{aligned}
\zeta_{+}^{\prime}(\varsigma) & :=\max \left\{0, \zeta^{\prime}(\varsigma)\right\}, \\
B_{1}\left(\varsigma, \varsigma_{1}\right) & :=\int_{\varsigma_{1}}^{\varsigma} \frac{d s}{r_{2}^{1 / \beta_{2}}(s)} \text { for } \varsigma \geq \varsigma_{1}, \\
B_{2}\left(\varsigma, \varsigma_{1}\right) & :=\left(\frac{B_{1}\left(\varsigma_{1}\right)}{r_{1}(\varsigma)}\right)^{1 / \beta_{1}} \text { for } \varsigma \geq \varsigma_{1}, \\
B_{3}\left(\varsigma, \varsigma_{2}\right) & :=\int_{\varsigma_{2}}^{\varsigma} B_{2}\left(s, \varsigma_{1}\right) d s \text { for } \varsigma \geq \varsigma_{2}>\varsigma_{1} .
\end{aligned}
$$

Throughout this paper, we assume that

$$
\psi_{1}(\varsigma):=\frac{1}{p\left(\varrho^{-1}(\varsigma)\right)}\left[1-\frac{1}{p\left(\varrho^{-1}\left(\varrho^{-1}(\varsigma)\right)\right)}\right]
$$

and

$$
\psi_{2}(\varsigma):=\frac{1}{p\left(\varrho^{-1}(\varsigma)\right)}\left[1-\frac{1}{p\left(\varrho^{-1}\left(\varrho^{-1}(\varsigma)\right)\right)} \frac{B_{3}\left(\varrho^{-1}\left(\varrho^{-1}(\varsigma)\right), \varsigma_{2}\right)}{B_{3}\left(\varrho^{-1}(\varsigma), \varsigma_{2}\right)}\right]
$$

for all sufficiently large $\varsigma$, where $\varrho^{-1}$ is the inverse function of $\varrho$, and we let

$$
\Omega_{1}(\varsigma):=\sum_{i=1}^{n} q_{i}(\varsigma)\left(\psi_{1}\left(\phi_{i}(\varsigma)\right)\right)^{\beta_{2}}, \quad \Omega_{2}(\varsigma):=\sum_{i=1}^{n} q_{i}(\varsigma)\left(\psi_{2}\left(\phi_{i}(\varsigma)\right)\right)^{\beta_{2}} .
$$

Lemma 1. If we let $x(\varsigma)$ be an eventually positive solution of (1), then $z(\varsigma)$ satisfies either $\left(C_{i}\right) z(\varsigma)>0, z^{\prime}(\varsigma)>0, r_{1}(\varsigma)\left(z^{\prime}(\varsigma)\right)^{\beta_{1}}>0$, and $r_{2}(\varsigma)\left(\left(r_{1}(\varsigma)\left(z^{\prime}(\varsigma)\right)^{\beta_{1}}\right)^{\prime}\right)^{\beta_{2}} \leq 0$, or $\left(C_{i i}\right) z(\varsigma)>0, z^{\prime}(\varsigma)<0, r_{1}(\varsigma)\left(z^{\prime}(\varsigma)\right)^{\beta_{1}}>0$, and $r_{2}(\varsigma)\left(\left(r_{1}(\varsigma)\left(z^{\prime}(\varsigma)\right)^{\beta_{1}}\right)^{\prime}\right)^{\beta_{2}} \leq 0$.

The proof of the above lemma is standard and so it is omitted.

Lemma 2. If (3) hold, and let $x(\varsigma)$ be an eventually positive solution of (1) with $z(\varsigma)$ satisfying $\left(C_{i i}\right)$ of Lemma 1. If

$$
\int_{\varsigma_{0}}^{\infty} \frac{1}{r_{1}^{1 / \beta_{1}}(v)}\left(\int_{v}^{\infty} \frac{1}{r_{2}^{1 / \beta_{2}}(u)}\left(\int_{u}^{\infty} \Omega_{1}(s) d s\right)^{1 / \beta_{2}} d u\right)^{1 / \beta_{1}} d v=\infty,
$$

then $\lim _{\zeta \rightarrow \infty} x(\varsigma)=0$.

Proof. Let $x(\varsigma)$ be an eventually positive solution of (1). Then, there exists $\varsigma_{1} \in\left[\varsigma_{0}, \infty\right)$ such that, for $\varsigma \geq \varsigma_{1}, x(\varsigma)>0, x(\varrho(\varsigma))>0, x\left(\phi_{i}(\varsigma)\right)>0$ for $i=1,2, \cdots, n$. From the definition of $z$, we obtain

$$
\begin{aligned}
x(\varsigma) & =\frac{1}{p\left(\varrho^{-1}(\varsigma)\right)}\left(z\left(\varrho^{-1}(\varsigma)\right)-x\left(\varrho^{-1}(\varsigma)\right)\right) \\
& =\frac{z\left(\varrho^{-1}(\varsigma)\right)}{p\left(\varrho^{-1}(\varsigma)\right)}-\frac{z\left(\varrho^{-1}\left(\varrho^{-1}(\varsigma)\right)\right)-x\left(\varrho^{-1}\left(\varrho^{-1}(\varsigma)\right)\right)}{p\left(\varrho^{-1}(\varsigma)\right) p\left(\varrho^{-1}\left(\varrho^{-1}(\varsigma)\right)\right)} \\
& \geq \frac{z\left(\varrho^{-1}(\varsigma)\right)}{p\left(\varrho^{-1}(\varsigma)\right)}-\frac{z\left(\varrho^{-1}\left(\varrho^{-1}(\varsigma)\right)\right)}{p\left(\varrho^{-1}(\varsigma)\right) p\left(\varrho^{-1}\left(\varrho^{-1}(\varsigma)\right)\right)} .
\end{aligned}
$$

From $\varrho(\varsigma)<\varsigma$, (iv) and the fact that $z(\varsigma)$ is decreasing, we have

$$
z\left(\varrho^{-1}(\varsigma)\right) \geq z\left(\varrho^{-1}\left(\varrho^{-1}(\varsigma)\right)\right) .
$$

Using this in (6), we obtain

$$
x(\varsigma) \geq \psi_{1}(\varsigma) z\left(\varrho^{-1}(\varsigma)\right),
$$


so

$$
x\left(\phi_{i}(\varsigma)\right) \geq \psi_{1}\left(\phi_{i}(\varsigma)\right) z\left(\varrho^{-1}\left(\phi_{i}(\varsigma)\right)\right), \quad i=1,2, \cdots, n .
$$

for $\varsigma \geq \varsigma_{2}$. Using (7) in (1) gives

$$
\left(r_{2}(\varsigma)\left(\left(r_{1}(\varsigma)\left(z^{\prime}(\varsigma)\right)^{\beta_{1}}\right)^{\prime}\right)^{\beta_{2}}\right)^{\prime}+\sum_{i=1}^{n} q_{i}(\varsigma)\left(\psi_{1}\left(\phi_{i}(\varsigma)\right)\right)^{\beta_{3}} z^{\beta_{3}}\left(\varrho^{-1}\left(\phi_{i}(\varsigma)\right)\right) \leq 0,
$$

for $\varsigma \geq \varsigma_{2}$. From (iv) $-(\mathrm{v})$ and the fact that $z(\varsigma)$ is decreasing, (8) yields

$$
\left.\left(r_{2}(\varsigma)\left(\left(r_{1}(\varsigma)\left(z^{\prime}(\varsigma)\right)^{\beta_{1}}\right)^{\prime}\right)^{\beta_{2}}\right)^{\prime}+z^{\beta_{3}}\left(\varrho^{-1}(\varsigma)\right)\right) \sum_{i=1}^{n} q_{i}(\varsigma)\left(\psi_{1}\left(\phi_{i}(\varsigma)\right)\right)^{\beta_{3}} \leq 0 \text { for } \varsigma \geq \varsigma_{2} \text {. }
$$

Since $z(\varsigma)>0$ and $z^{\prime}(\varsigma)<0$, there exists a constant $\kappa$, such that

$$
\lim _{\zeta \rightarrow \infty} z(\varsigma)=\kappa<\infty
$$

where $\kappa \geq 0$. If $\kappa>0$, then there exists $\varsigma_{3} \geq \varsigma_{2}$, such that $\varrho^{-1}\left(\theta_{1}(\varsigma)\right)>\varsigma_{2}$ and

$$
z(\varsigma) \geq \kappa \text { for } \varsigma \geq \varsigma_{3}
$$

Integrating (9) from $\varsigma$ to $\infty$ two times gives

$$
\left.-z^{\prime}(\varsigma) \geq \kappa^{\frac{\beta_{3}}{\beta_{2}}} \frac{1}{r_{1}^{1 / \beta_{1}}(s)}\left(\int_{\varsigma}^{\infty} \frac{1}{r_{2}^{1 / \beta_{2}}(u)}\left(\int_{u}^{\infty} \Omega_{1}(s) d s\right)^{1 / \beta_{2}}\right)^{1 / \beta_{2}} d u\right)^{1 / \beta_{1}} .
$$

Integrating the resulting inequality from $\varsigma_{3}$ to $\varsigma$ yields

$$
z\left(\varsigma_{3}\right) \geq \kappa^{\frac{\beta_{3}}{\beta_{1} \beta_{2}}} \int_{\varsigma_{0}}^{\infty} \frac{1}{r_{1}^{1 / \beta_{1}}(v)}\left(\int_{v}^{\infty} \frac{1}{r_{2}^{1 / \beta_{2}}(u)}\left(\int_{u}^{\infty} \Omega_{1}(s) d s\right)^{1 / \beta_{2}} d u\right)^{1 / \beta_{1}} d v
$$

which contradicts (5), and so we have $\kappa=0$. Therefore, $\lim _{\zeta \rightarrow \infty} z(\varsigma)=0$. Since $0<x(\varsigma) \leq$ $z(\varsigma)$ on $\left[\varsigma_{1}, \infty\right)$, we obtain $\lim _{\varsigma \rightarrow \infty} x(\varsigma)=0$.

Now, we are ready to present our main results. We now establish the oscillation criteria for $(1)$ in the case $\varrho(\zeta) \geq \phi_{i}(\varsigma)$.

Theorem 1. If $\varrho(\varsigma) \geq \phi_{i}(\varsigma)$ for $i=1,2, \cdots, n$ and $\psi_{1}(\varsigma)>0, \psi_{2}(\varsigma)>0$, (5) hold. If there exists a function $\zeta \in C^{1}\left(\left[\varsigma_{0}, \infty\right), \mathbb{R}\right)$ and $\eta \in C^{1}\left(\left[\varsigma_{0}, \infty\right), \mathbb{R}\right)$ such that $r_{2} \eta \in C^{1}\left(\left[\varsigma_{0}, \infty\right), \mathbb{R}\right)$ and

$$
\limsup _{s \rightarrow \infty} \int_{T}^{\varsigma}\left\{\Phi(s)-\frac{\delta\left(\zeta_{+}^{\prime}(s)+\beta_{3}\left(1+\frac{1}{\beta_{1} \beta_{2}}\right) \zeta(s) \varphi(s) B_{2}\left(s, s_{1}\right)\left(r_{2}(s) \eta(s)\right)^{\frac{1}{\beta_{1} \beta_{2}}}\right)^{\beta_{1} \beta_{2}+1}}{\left(\zeta(s) \varphi(s) B_{2}\left(s, s_{1}\right)\right)^{\beta_{1} \beta_{2}}}\right\} d s=\infty
$$

where

$$
\begin{aligned}
\Phi(\varsigma)=\zeta(\varsigma) \sum_{i=1}^{n} q_{i}(\varsigma)\left(\psi_{2}\left(\phi_{i}(\varsigma)\right)\right)^{\beta_{3}}\left(\frac{B_{3}\left(\varrho^{-1}\left(\phi_{i}(\varsigma)\right), \varsigma_{2}\right)}{B_{3}(\varsigma, \varsigma 2)}\right)^{\beta_{3}} \\
\quad+\frac{\beta_{3}}{\beta_{1} \beta_{2}} \zeta(\varsigma) \varphi(\varsigma) B_{2}\left(\varsigma, \varsigma_{1}\right)\left(r_{2}(\varsigma) \eta(\varsigma)\right)^{\left(1+\frac{1}{\beta_{1} \beta_{2}}\right)}-\zeta(\varsigma)\left(r_{2}(\varsigma) \eta(\varsigma)\right)^{\prime}, \\
\varphi(\varsigma)= \begin{cases}m_{1}, & m_{1} \text { is any positive constant; if } \beta_{1} \beta_{2} \leq \beta_{3}, \\
m_{2}\left(B_{3}\left(\varsigma, \varsigma_{1}\right)\right)^{\frac{\beta_{3}}{\beta_{1} \beta_{2}}-1}, & m_{2} \text { is any positive constant; if } \beta_{1} \beta_{2}>\beta_{3},\end{cases} \\
\delta=\left(\frac{\beta_{1} \beta_{2}}{\beta_{3}}\right)^{\beta_{1} \beta_{2}}\left(\frac{1}{\beta_{1} \beta_{2}+1}\right)^{\beta_{1} \beta_{2}+1},
\end{aligned}
$$


for all $\varsigma_{1}, \varsigma_{2}, T \in\left[\varsigma_{0}, \infty\right)$, where $T>\varsigma_{2}>\varsigma_{1}$, then any solution of (1) is either oscillatory or satisfies $\lim _{\varsigma \rightarrow \infty} x(\varsigma)=0$.

Proof. Let (1) have a nonoscillatory solution $x(\varsigma)$ on $\left[\varsigma_{0}, \infty\right)$-say there exists $\varsigma_{1} \in\left[\varsigma_{0}, \infty\right)$ such that, for $\varsigma \geq \varsigma_{1}, x(\varsigma)>0, x(\varrho(\varsigma))>0$, and $x\left(\phi_{i}(\varsigma)\right)>0$, (3) and (4) hold, and $z(\varsigma)$ satisfies either $\left(C_{i}\right)$ or $\left(C_{i i}\right)$ and $i=1,2, \cdots, n$. Assume that $\left(C_{i}\right)$ holds, proceeding as in the proof of Lemma 2, we obtain (6). Since $r_{2}(\varsigma)\left(\left(r_{1}(\varsigma)\left(z^{\prime}(\varsigma)\right)^{\beta_{1}}\right)^{\prime}\right)^{\beta_{2}}$ is decreasing, we see that

$$
\begin{aligned}
r_{1}(\varsigma)\left(z^{\prime}(\varsigma)\right)^{\beta_{1}} & =r_{1}\left(\varsigma_{1}\right)\left(z^{\prime}\left(\varsigma_{1}\right)\right)^{\beta_{1}}+\int_{\varsigma_{1}}^{\varsigma} \frac{r_{2}^{1 / \beta_{2}}(s)\left(r_{1}(s)\left(z^{\prime}(s)\right)^{\beta_{1}}\right)^{\prime}}{r_{2}^{-1 / \beta_{2}}(s)} d s \\
& \geq r_{2}^{1 / \beta_{2}}(\varsigma)\left(r_{1}(\varsigma)\left(z^{\prime}(\varsigma)\right)^{\beta_{1}}\right)^{\prime} B_{1}\left(\varsigma, \varsigma_{1}\right) \quad \text { for } \varsigma \geq \varsigma_{1} .
\end{aligned}
$$

From (12), we have for all $\varsigma \geq \varsigma_{2}:=\varsigma_{1}+1$ that

$$
\left(r_{1}(\varsigma)\left(z^{\prime}(\varsigma)\right)^{\beta_{1}}\right)^{\prime}=\frac{r_{2}^{-1 / \beta_{2}}(\varsigma)\left[r_{2}^{1 / \beta_{2}}(\varsigma)\left(r_{1}(\varsigma)\left(z^{\prime}(\varsigma)\right)^{\beta_{1}}\right)^{\prime} B_{1}\left(\varsigma, \varsigma_{1}\right)-r_{1}(\varsigma)\left(z^{\prime}(\varsigma)\right)^{\beta_{1}}\right]}{\left(B_{1}\left(\varsigma, \varsigma_{1}\right)\right)^{2}} \leq 0,
$$

so $z^{\prime}(\varsigma) / B_{1}\left(\varsigma, \varsigma_{1}\right)$ is decreasing for $\varsigma \geq \varsigma_{2}$. Next, using that $z^{\prime}(\varsigma) / B_{1}\left(\varsigma, \varsigma_{1}\right)$ is decreasing for $\varsigma \geq \varsigma_{2}$, we obtain

$$
\begin{aligned}
z(\varsigma) & =z\left(\varsigma_{2}\right)+\int_{\varsigma_{2}}^{\varsigma} \frac{z^{\prime}(s)}{B_{2}\left(s, \varsigma_{1}\right)} B_{2}\left(s_{,} \varsigma_{1}\right) d s \\
& \geq \frac{B_{3}\left(\varsigma, \varsigma_{2}\right)}{B_{2}\left(\varsigma, \varsigma_{1}\right)} z^{\prime}(\varsigma) \quad \text { for } \varsigma \geq \varsigma_{2} .
\end{aligned}
$$

From (13), for all $\varsigma \geq \varsigma_{3}:=\varsigma_{2}+1$, we have that

$$
\left(\frac{z(\varsigma)}{B_{3}\left(\varsigma, \varsigma_{2}\right)}\right)^{\prime}=\frac{z^{\prime}(\varsigma) B_{3}\left(\varsigma_{,} \varsigma_{2}\right)-z(\varsigma) B_{2}\left(\varsigma_{,} \varsigma_{1}\right)}{\left(B_{3}\left(\varsigma, \varsigma_{2}\right)\right)^{2}} \leq 0,
$$

so $z(\varsigma) / B_{3}\left(\varsigma, \varsigma_{2}\right)$ is decreasing for $\varsigma \geq \varsigma_{3}$. Next, in view of the fact that $z(\varsigma) / B_{3}\left(\varsigma, \varsigma_{2}\right)$ is decreasing for $\varsigma \geq \varsigma_{3}$ and $\varrho(\varsigma)<\varsigma$ or $\varrho^{-1}(\varsigma) \leq \varrho^{-1}\left(\varrho^{-1}(\varsigma)\right)$, we obtain

$$
\frac{B_{3}\left(\varrho^{-1}\left(\varrho^{-1}(\varsigma)\right), \varsigma_{2}\right) z\left(\varrho^{-1}(\varsigma)\right)}{B_{3}\left(\varrho^{-1}(\varsigma), \varsigma_{2}\right)} \geq z\left(\varrho^{-1}\left(\varrho^{-1}(\varsigma)\right)\right)
$$

Using (15) in (6) yields

$$
x(\varsigma) \geq \frac{1}{p\left(\varrho^{-1}(\varsigma)\right)}\left[1-\frac{1}{p\left(\varrho^{-1}\left(\varrho^{-1}(\varsigma)\right)\right)} \frac{B_{3}\left(\varrho^{-1}\left(\varrho^{-1}(\varsigma)\right), \varsigma_{2}\right)}{B_{3}\left(\varrho^{-1}(\varsigma), \varsigma_{2}\right)}\right] z\left(\varrho^{-1}(\varsigma)\right)=\psi_{2}(\varsigma) z\left(\varrho^{-1}(\varsigma)\right),
$$

so

$$
x\left(\phi_{i}(\varsigma)\right) \geq \psi_{2}\left(\phi_{i}(\varsigma)\right) z\left(\varrho^{-1}\left(\phi_{i}(\varsigma)\right)\right), \quad i=1,2, \cdots, n
$$

for $\varsigma \geq \varsigma_{3}$. Using (16) in (1) gives

$$
\left(r_{2}(\varsigma)\left(\left(r_{1}(\varsigma)\left(z^{\prime}(\varsigma)\right)^{\beta_{1}}\right)^{\prime}\right)^{\beta_{2}}\right)^{\prime}+\sum_{i=1}^{n} q_{i}(\varsigma)\left(\psi_{2}\left(\phi_{i}(\varsigma)\right)\right)^{\beta_{3}} z^{\beta_{3}}\left(\varrho^{-1}\left(\phi_{i}(\varsigma)\right)\right) \leq 0 .
$$

Define

$$
w(\varsigma)=\zeta(\varsigma)\left[\frac{r_{2}(\varsigma)\left(\left(r_{1}(\varsigma)\left(z^{\prime}(\varsigma)\right)^{\beta_{1}}\right)^{\prime}\right)^{\beta_{2}}}{\left(z^{\prime}(\varsigma)\right)^{\beta_{3}}}+r_{2}(\varsigma) \eta(\varsigma)\right] \quad \text { for } \varsigma \geq \varsigma_{1} .
$$


Then $w(\varsigma)>0$, and from (17), we see that

$$
\begin{aligned}
w^{\prime}(\varsigma)= & \zeta^{\prime}(\varsigma)\left[\frac{r_{2}(\varsigma)\left(\left(r_{1}(\varsigma)\left(z^{\prime}(\varsigma)\right)^{\beta_{1}}\right)^{\prime}\right)^{\beta_{2}}}{\left(z^{\prime}(\varsigma)\right)^{\beta_{3}}}+r_{2}(\varsigma) \eta(\varsigma)\right] \\
& +\zeta(\varsigma)\left[\frac{r_{2}(\varsigma)\left(\left(r_{1}(\varsigma)\left(z^{\prime}(\varsigma)\right)^{\beta_{1}}\right)^{\prime}\right)^{\beta_{2}}}{\left(z^{\prime}(\varsigma)\right)^{\beta_{3}}}+r_{2}(\varsigma) \eta(\varsigma)\right]^{\prime} \\
\leq & \frac{\zeta^{\prime}(\varsigma)}{\zeta(\varsigma)} w(\varsigma)+\zeta(\varsigma)\left(r_{2}(\varsigma) \eta(\varsigma)\right)^{\prime}-\zeta(\varsigma)\left[\sum_{i=1}^{n} q_{i}(s)\left(\psi_{2}\left(\phi_{i}(s)\right)\right)^{\beta_{3}} \frac{z^{\beta_{2}}\left(\varrho^{-1}\left(\phi_{i}(\varsigma)\right)\right)}{\left(z^{\prime}(\varsigma)\right)^{\beta_{2}}}\right] \\
& -\beta_{3} \zeta(\varsigma) r_{2}(\varsigma) \frac{r_{2}(\varsigma)\left(\left(r_{1}(\varsigma)\left(z^{\prime}(\varsigma)\right)^{\beta_{1}}\right)^{\prime}\right)^{\beta_{2}} z^{\prime}(\varsigma)}{\left(z^{\prime}(\varsigma)\right)^{\beta_{3}+1}} .
\end{aligned}
$$

Using the fact $z(\varsigma) / B_{3}\left(\varsigma_{,} \varsigma_{2}\right)$ is nonincreasing for $\varsigma \geq \varsigma_{3}$, and noting that $\varrho(\varsigma) \geq \phi_{i}(\varsigma)$ implies $\varrho^{-1}\left(\phi_{i}(\varsigma)\right) \leq \varsigma$, we obtain

$$
\frac{z\left(\varrho^{-1}\left(\phi_{i}(\varsigma)\right)\right)}{z(\varsigma)} \geq \frac{B_{3}\left(\varrho^{-1}\left(\phi_{i}(\varsigma)\right), \varsigma_{2}\right)}{B_{3}\left(\varsigma, \varsigma_{2}\right)}, \quad i=1,2, \cdots, n
$$

for $\varsigma \geq \varsigma_{3}$. Substituting (13), (18) and (20) into (19), we obtain

$$
\begin{gathered}
w^{\prime}(\varsigma) \leq \frac{\zeta^{\prime}(\varsigma)}{\zeta(\varsigma)} w(\varsigma)+\zeta(\varsigma)\left(r_{2}(\varsigma) \eta(\varsigma)\right)^{\prime}-\zeta(\varsigma)\left[\sum_{i=1}^{n} q_{i}(\varsigma)\left(\psi_{2}\left(\phi_{i}(\varsigma)\right)\right)^{\beta_{3}}\left(\frac{B_{3}\left(\varrho^{-1}\left(\phi_{i}(\varsigma)\right), \varsigma_{2}\right)}{B_{3}\left(\varsigma, \varsigma_{1}\right)}\right)^{\beta_{3}}\right] \\
-\beta_{3} \zeta(\varsigma) B_{2}\left(\varsigma, \varsigma_{1}\right)(z(\varsigma))^{\frac{\beta_{3}}{\beta_{1} \beta_{2}}-1}\left(\frac{w(\varsigma)}{\zeta(\varsigma)}-r_{2}(\varsigma) \eta(\varsigma)\right)^{1+\frac{1}{\beta_{1} \beta_{2}}} . \quad \text { for } \varsigma \geq \varsigma_{3} .
\end{gathered}
$$

Next, we will compute $z^{\frac{\beta_{3}}{\beta_{1} \beta_{2}}-1}(\varsigma)$ and consider the following two cases:

(1) $\beta_{1} \beta_{2} \leq \beta_{3}$. From $z^{\prime}(\varsigma)>0$, there exists a constant $h_{1}>0$, such that

$$
z(\varsigma) \geq z\left(\varsigma_{1}\right)=h_{1} \quad \text { for } \varsigma \geq \varsigma_{2},
$$

which implies that

$$
z^{\frac{\beta_{3}}{\beta_{1} \beta_{2}}-1}(\varsigma) \geq h_{1}^{\frac{\beta_{3}}{\beta_{1} \beta_{2}}-1}:=m_{1}, \quad \text { for } \varsigma \geq \varsigma_{2} .
$$

(2) $\beta_{1} \beta_{2}>\beta_{3}$. From (14), there exists a constant $h_{2}>0$ and $\varsigma_{3} \geq \varsigma_{2}$, such that

$$
\frac{z(\varsigma)}{B_{3}\left(\varsigma_{1} \varsigma_{1}\right)} \leq \frac{z(\varsigma)}{B_{3}\left(\varsigma_{2}, \varsigma_{1}\right)}=h_{2} \quad \text { for } \varsigma \geq \varsigma_{3}
$$

Hence,

$$
\begin{gathered}
z^{\frac{\beta_{3}}{\beta_{1} \beta_{2}}-1}(\varsigma) \geq h_{2}^{\frac{\beta_{3}}{\beta_{1} \beta_{2}}-1}\left(B_{3}\left(\varsigma, \varsigma_{1}\right)\right)^{\frac{\beta_{3}}{\beta_{1} \beta_{2}}-1}=m_{2}\left(B_{3}\left(\varsigma, \varsigma_{1}\right)\right)^{\frac{\beta_{3}}{\beta_{1} \beta_{2}}-1} \text { for } \varsigma \geq \varsigma_{2}, \\
\text { where } m_{2}=h_{2}^{\frac{\beta_{3}}{\beta_{1} \beta_{2}}-1} \cdot \\
\text { Combining }(21) \text { with (22) and (23), we obtain } \\
w^{\prime}(\varsigma) \leq \frac{\zeta^{\prime}(\varsigma)}{\zeta(\varsigma)} w(\varsigma)+\zeta(\varsigma)\left(r_{2}(\varsigma) \eta(\varsigma)\right)^{\prime}-\zeta(\varsigma)\left[\sum_{i=1}^{n} q_{i}(\varsigma)\left(\psi_{2}\left(\phi_{i}(\varsigma)\right)\right)^{\beta_{3}}\left(\frac{B_{3}\left(\varrho^{-1}\left(\phi_{i}(\varsigma)\right), \varsigma_{2}\right)}{B_{3}\left(\varsigma, \varsigma_{1}\right)}\right)^{\beta_{3}}\right] \\
-\beta_{3} \zeta(\varsigma) \varphi(\varsigma) B_{2}(\varsigma, \varsigma 1)\left(\frac{w(\varsigma)}{\zeta(\varsigma)}-r_{2}(\varsigma) \eta(\varsigma)\right)^{1+\frac{1}{\beta_{1} \beta_{2}}} . \text { for } \varsigma \geq \varsigma_{3} .
\end{gathered}
$$


By applying the inequality

$$
X^{1+\frac{1}{\alpha}}-(X-Y)^{1+\frac{1}{\alpha}} \leq Y^{\frac{1}{\alpha}}\left[\left(1+\frac{1}{\alpha}\right) X-\frac{1}{\alpha} Y\right] \quad \text { for } \quad \alpha=\text { odd } / \text { odd } \geq 1,
$$

we obtain

$$
\begin{aligned}
\left(\frac{w(\varsigma)}{\zeta(\varsigma)}-r_{2}(\varsigma) \eta(\varsigma)\right)^{1+\frac{1}{\beta_{1} \beta_{2}}} \geq\left(\frac{w(\varsigma)}{\zeta(\varsigma)}\right)^{1+\frac{1}{\beta_{1} \beta_{2}}}+\frac{1}{\beta_{1} \beta_{2}}\left(r_{2}(\varsigma) \eta(\varsigma)\right)^{1+\frac{1}{\beta_{1} \beta_{2}}} \\
-\left(1+\frac{1}{\beta_{1} \beta_{2}}\right) \frac{\left(r_{2}(\varsigma) \eta(\varsigma)\right)^{\frac{1}{\beta_{1} \beta_{2}}}}{\zeta(\varsigma)} \omega(\varsigma)
\end{aligned}
$$

Substituting (24) into (25), we see that

$$
\begin{aligned}
& w^{\prime}(\varsigma) \leq \frac{\zeta^{\prime}(\varsigma)}{\zeta(\varsigma)} w(\varsigma)+\zeta(\varsigma)\left(r_{2}(\varsigma) \eta(\varsigma)\right)^{\prime}-\zeta(\varsigma)\left[\sum_{i=1}^{n} q_{i}(\varsigma)\left(\psi_{2}\left(\phi_{i}(\varsigma)\right)\right)^{\beta_{3}}\left(\frac{B_{3}\left(\varrho^{-1}\left(\phi_{i}(\varsigma)\right), \varsigma_{2}\right)}{B_{3}\left(\varsigma, \varsigma_{1}\right)}\right)^{\beta_{3}}\right] \\
& -\frac{\beta_{3}}{\beta_{1} \beta_{2}} \zeta(\varsigma) \varphi(\varsigma) B_{2}\left(\varsigma, \varsigma_{1}\right)\left(r_{2}(\varsigma) \eta(\varsigma)\right)^{1+\frac{1}{\beta_{1} \beta_{2}}}-\beta_{3} \frac{\varphi(\varsigma) B_{2}\left(\varsigma, \varsigma_{1}\right)}{(\zeta(\varsigma))^{\frac{1}{\beta_{1} \beta_{2}}}}(w(\varsigma))^{1+\frac{1}{\beta_{1} \beta_{2}}} \\
& +\left(1+\frac{1}{\beta_{1} \beta_{2}}\right) \beta_{3} \varphi(\varsigma) B_{2}\left(\varsigma, \varsigma_{1}\right)\left(r_{2}(\varsigma) \eta(\varsigma)\right)^{\frac{1}{\beta_{1} \beta_{2}}} \omega(\varsigma) \\
& =\zeta(\varsigma)\left(r_{2}(\varsigma) \eta(\varsigma)\right)^{\prime}-\zeta(\varsigma)\left[\sum_{i=1}^{n} q_{i}(\varsigma)\left(\psi_{2}\left(\phi_{i}(\varsigma)\right)\right)^{\beta_{3}}\left(\frac{B_{3}\left(\varrho^{-1}\left(\phi_{i}(\varsigma)\right), \varsigma_{2}\right)}{B_{3}\left(\varsigma, \varsigma_{1}\right)}\right)^{\beta_{3}}\right] \\
& -\frac{\beta_{3}}{\beta_{1} \beta_{2}} \zeta(\varsigma) \varphi(\varsigma) B_{2}\left(\varsigma, \varsigma_{1}\right)\left(r_{2}(\varsigma) \eta(\varsigma)\right)^{1+\frac{1}{\beta_{1} \beta_{2}}} \\
& +\left\{\frac{\zeta_{+}^{\prime}(\varsigma)}{\zeta(\varsigma)}+\left(1+\frac{1}{\beta_{1} \beta_{2}}\right) \beta_{3} \varphi(\varsigma) B_{2}\left(\varsigma, \varsigma_{1}\right)\left(r_{2}(\varsigma) \eta(\varsigma)\right)^{\frac{1}{\beta_{1} \beta_{2}}}\right\} \omega(\varsigma) \\
& -\beta_{3} \frac{\varphi(\varsigma) B_{2}\left(\varsigma, \varsigma_{1}\right)}{(\zeta(\varsigma))^{\frac{1}{\beta_{1} \beta_{2}}}}(w(\varsigma))^{1+\frac{1}{\beta_{1} \beta_{2}}} \\
& =-\Phi(\varsigma)+C(\varsigma) \omega(\varsigma)-D(\varsigma)(w(\varsigma))^{1+\frac{1}{\beta_{1} \beta_{2}}},
\end{aligned}
$$

where

$$
\begin{aligned}
& C(\varsigma):=\frac{\zeta_{+}^{\prime}(\varsigma)}{\zeta(\varsigma)}+\left(1+\frac{1}{\beta_{1} \beta_{2}}\right) \beta_{3} \varphi(\varsigma) B_{2}\left(\varsigma, \varsigma_{1}\right)\left(r_{2}(\varsigma) \eta(\varsigma)\right)^{\frac{1}{\beta_{1} \beta_{2}}}, \\
& D(\varsigma):=\beta_{3} \frac{\varphi(\varsigma) B_{2}\left(\varsigma, \varsigma_{1}\right)}{(\zeta(\varsigma))^{\frac{1}{\beta_{1} \beta_{2}}}}
\end{aligned}
$$

for $\varsigma \geq \varsigma_{3}$. Using the inequality

$$
C \omega-D \omega^{1+1 / \alpha} \leq \frac{\alpha^{\alpha}}{(\alpha+1)^{\alpha+1}} \frac{C^{\alpha+1}}{D^{\alpha}}
$$

$D>0$, we obtain

$$
w^{\prime}(\varsigma) \leq-\Phi(\varsigma)+\frac{\delta\left(\zeta_{+}^{\prime}(\varsigma)+\beta_{3}\left(1+\frac{1}{\beta_{1} \beta_{2}}\right) \zeta(\varsigma) \varphi(\varsigma) B_{2}\left(\varsigma, \varsigma_{0}\right)\left(r_{2}(\varsigma) \eta(\varsigma)\right)^{\frac{1}{\beta_{1} \beta_{2}}}\right)^{\beta_{1} \beta_{2}+1}}{\left(\zeta(\varsigma) \varphi(\varsigma) B_{2}\left(\varsigma, \varsigma_{0}\right)\right)^{\beta_{1} \beta_{2}}} .
$$

An integration of (28) from $\varsigma_{3}$ to $\varsigma$ yields 


$$
\int_{T}^{s}\left\{\Phi(s)-\frac{\delta\left(\zeta_{+}^{\prime}(s)+\beta_{3}\left(1+\frac{1}{\beta_{1} \beta_{2}}\right) \zeta(s) \varphi(s) B_{2}\left(s, \varsigma_{0}\right)\left(r_{2}(s) \eta(s)\right)^{\frac{1}{\beta_{1} \beta_{2}}}\right)^{\beta_{1} \beta_{2}+1}}{\left(\zeta(s) \varphi(s) B_{2}\left(s, \varsigma_{0}\right)\right)^{\beta_{1} \beta_{2}}}\right\} d s<w\left(\varsigma_{2}\right)
$$

which contradicts (11).

Assume that $\left(C_{i i}\right)$ holds, by Lemma 2, we have $\lim _{\zeta \rightarrow \infty} x(\varsigma)=0$. The proof is complete.

Next, we examine the oscillation results of the solutions of (1) by Philos-type [9]. Let $\mathbb{S}_{0}=\{(\varsigma, s): a \leq s<\varsigma<+\infty\}, \mathbb{S}=\{(\varsigma, s): a \leq s \leq \varsigma<+\infty\}$ the continuous function $E(\varsigma, s), E: \mathbb{S} \rightarrow \mathbb{R}$ belongs to the class function $\Re$

$\left(C_{i}\right) E(\varsigma, \varsigma)=0$ for $\varsigma \geq \varsigma_{0}$ and $E(\varsigma, s)>0$ for $(\varsigma, s) \in \mathbb{S}_{0}$,

$\left(C_{i i}\right) \frac{\partial E(\varsigma, s)}{\partial s} \leq 0,(\varsigma, s) \in \mathbb{S}_{0}$ and some locally integrable function $e(\varsigma, s)$, such that

$$
\frac{\partial E(\varsigma, s)}{\partial s}+E(\varsigma, s)\left[\frac{\zeta_{+}^{\prime}(\varsigma)}{\zeta(\varsigma)}+\beta_{3}\left(1+\frac{1}{\beta_{1} \beta_{2}}\right) \varphi(\varsigma) B_{2}\left(\varsigma, \varsigma_{0}\right)\left(r_{2}(\varsigma) \eta(\varsigma)\right)^{\frac{1}{\beta_{1} \beta_{2}}}\right]=-e(\varsigma, s)
$$

for all $(\varsigma, s) \in \mathbb{S}_{0}$.

Theorem 2. If $\varrho(\varsigma) \geq \phi_{i}(\varsigma)$ for $i=1,2, \cdots, n$ and $\psi_{1}(\varsigma)>0, \psi_{2}(\varsigma)>0$, (5) holds. If there exists a function $\zeta \in C^{1}\left(\left[\varsigma_{0}, \infty\right), \mathbb{R}\right)$ and $\eta \in C^{1}\left(\left[\varsigma_{0}, \infty\right), \mathbb{R}\right)$ such that $r_{2} \eta \in C^{1}\left(\left[\varsigma_{0}, \infty\right), \mathbb{R}\right)$ and

$$
\limsup _{\varsigma \rightarrow \infty} \frac{1}{E\left(\varsigma, \varsigma_{*}\right)} \int_{T}^{\varsigma}\left\{E(\varsigma, s) \Phi(s)-\frac{\delta \zeta(s)|e(\varsigma, s)|^{\beta_{1} \beta_{2}+1}}{\left(E(\varsigma, s) \varphi(s) B_{2}\left(s, \varsigma_{1}\right)\right)^{\beta_{1} \beta_{2}}}\right\} d s=\infty,
$$

for all $\varsigma_{1}, \varsigma_{2}, \varsigma_{*} \in\left[\varsigma_{0}, \infty\right)$, where $\delta, \Phi(\varsigma), \varphi(\varsigma)$ are defined as in Theorem 1 and $\varsigma_{*}>\varsigma_{2}>\varsigma_{1}$, then any solution of (1) is either oscillatory or satisfies $\lim _{\varsigma \rightarrow \infty} x(\varsigma)=0$.

Proof. Let (1) have a nonoscillatory solution $x(\varsigma)$ on $\left[\varsigma_{0}, \infty\right)$-say there exists $\varsigma_{1} \in\left[\varsigma_{0}, \infty\right)$ such that, for $\varsigma \geq \varsigma_{1}, x(\varsigma)>0, x(\varrho(\varsigma))>0$, and $x\left(\phi_{i}(\varsigma)\right)>0$, (3) and (4) hold, and $z(\varsigma)$ satisfies either $\left(C_{i}\right)$ or $\left(C_{i i}\right)$ and $i=1,2, \cdots, n$. Assume that $\left(C_{i}\right)$ holds. Following the same arguments as in the proof of Theorem 1, we obtain (26). In view of (18), inequality (26) takes the form

$$
\Phi(\varsigma) \leq-w^{\prime}(\varsigma)+C(\varsigma) \omega(\varsigma)-D(\varsigma)(w(\varsigma))^{1+\frac{1}{\beta_{1} \beta_{2}}}
$$

Multiplying $E(\varsigma, s)$ integrating (30) from $\varsigma_{3}$ to $\varsigma$, one can obtain

$$
\begin{aligned}
& \int_{\varsigma_{3}}^{\varsigma} E(\varsigma, s) \Phi(s) d s \leq-\int_{\varsigma_{3}}^{\varsigma} E(\varsigma, s) w^{\prime}(s) d s+\int_{\varsigma_{3}}^{\varsigma} E(\varsigma, s) C(s) \omega(s) d s-\int_{\zeta_{3}}^{\varsigma} E(\varsigma, s) D(s)(w(s))^{1+\frac{1}{\beta_{1} \beta_{2}}} d s \\
& =E\left(\varsigma_{,} \varsigma_{3}\right) \omega\left(\varsigma_{3}+\int_{\varsigma_{3}}^{\varsigma}\left[\frac{\partial E(\varsigma, s)}{\partial s}+E(\varsigma, s) C(s)\right] \omega(s) d s-\int_{\varsigma_{3}}^{\varsigma} E(\varsigma, s) D(s)(w(s))^{1+\frac{1}{\beta_{1} \beta_{2}}} d s\right. \\
& =E\left(\varsigma_{1} \varsigma_{3}\right) \omega\left(\varsigma_{3}+\int_{\varsigma_{3}}^{\varsigma} e(\varsigma, s) \omega(s) d s-\int_{\varsigma_{3}}^{\varsigma} E(\varsigma, s) D(s)(w(s))^{1+\frac{1}{\beta_{1} \beta_{2}}} d s\right. \\
& \leq E\left(\varsigma_{,} \varsigma_{3}\right) \omega\left(\varsigma_{3}+\int_{\varsigma_{3}}^{\varsigma}\left\{|e(\varsigma, s)| \omega(s)-E(\varsigma, s) D(s)(w(s))^{1+\frac{1}{\beta_{1} \beta_{2}}}\right\} d s\right.
\end{aligned}
$$

Now, using the inequality (27), we obtain

$$
\int_{\varsigma_{3}}^{\varsigma} E(\varsigma, s) \Phi(s) d s \leq E\left(\varsigma, \varsigma_{3}\right) \omega\left(\varsigma_{3}\right)+\int_{\varsigma_{3}}^{\varsigma} \frac{\delta \zeta(s)|e(\varsigma, s)|^{\beta_{1} \beta_{2}+1}}{\left(E(\varsigma, s) \varphi(s) B_{2}\left(s, \varsigma_{1}\right)\right)^{\beta_{1} \beta_{2}}} d s,
$$


and this implies that

$$
\frac{1}{E\left(\varsigma, \varsigma_{*}\right)} \int_{T}^{\varsigma}\left\{E(\varsigma, s) \Phi(s)-\frac{\delta \zeta(s)|e(\varsigma, s)|^{\beta_{1} \beta_{2}+1}}{\left(E(\varsigma, s) \varphi(s) B_{2}\left(s, \varsigma_{1}\right)\right)^{\beta_{1} \beta_{2}}}\right\} d s \leq w(T),
$$

which contradicts (29).

Suppose that $\left(C_{i i}\right)$ holds, and so $\lim _{\varsigma \rightarrow \infty} x(\varsigma)=0$ by Lemma 2 . The proof is complete.

Corollary 1. Suppose that all conditions of Theorem 2 are satisfied with (29) replaced by

$$
\limsup _{\varsigma \rightarrow \infty} \frac{1}{E\left(\varsigma, \varsigma_{*}\right)} \int_{\varsigma_{*}}^{\varsigma} E(\varsigma, s) \Phi(s) d s=\infty
$$

and

$$
\limsup _{\zeta \rightarrow \infty} \frac{1}{E\left(\varsigma, \varsigma_{*}\right)} \int_{\zeta_{*}}^{\varsigma} \frac{\delta \zeta(\varsigma)|e(\zeta, s)|^{\beta_{1} \beta_{2}+1}}{\left(E(\varsigma, s) \varphi(s) B_{2}\left(s, \varsigma_{1}\right)\right)^{\beta_{1} \beta_{2}}} d s<\infty
$$

then any solution of (1) is either oscillatory or satisfies $\lim _{\zeta \rightarrow \infty} x(\varsigma)=0$.

Next, we establish the oscillation criteria for $(1)$ in the case $\varrho(\varsigma) \leq \phi_{i}(\varsigma)$.

Theorem 3. If $\varrho(\varsigma) \leq \phi_{i}(\varsigma)$ for $i=1,2, \cdots, n$ and $\psi_{1}(\varsigma)>0, \psi_{2}(\varsigma)>0$, (5) holds. If there exists a function $\zeta \in C^{1}\left(\left[\varsigma_{0}, \infty\right), \mathbb{R}\right)$ and $\eta \in C^{1}\left(\left[\varsigma_{0}, \infty\right), \mathbb{R}\right)$ such that $r_{2} \eta \in C^{1}\left(\left[\varsigma_{0}, \infty\right), \mathbb{R}\right)$ and

$$
\begin{gathered}
\limsup _{s \rightarrow \infty} \int_{T}^{\zeta}\left\{\Phi_{*}(s)-\frac{\delta\left(\zeta_{+}^{\prime}(s)+\beta_{3}\left(1+\frac{1}{\beta_{1} \beta_{2}}\right) \zeta(s) \varphi(s) B_{2}\left(s, \varsigma_{0}\right)\left(r_{2}(s) \eta(s)\right)^{\frac{1}{\beta_{1} \beta_{2}}}\right)^{\beta_{1} \beta_{2}+1}}{\left(\zeta(s) \varphi(s) B_{2}\left(s, \varsigma_{0}\right)\right)^{\beta_{1} \beta_{2}}}\right\} d s=\infty, \\
\text { where } \\
\Phi_{*}(\varsigma)=\zeta(s) \sum_{i=1}^{n} q_{i}(s)\left(\psi_{2}\left(\phi_{i}(s)\right)\right)^{\beta_{3}}-\zeta(s)\left(r_{2}(s) \eta(s)\right)^{\prime} \\
+\frac{\beta_{3}}{\beta_{1} \beta_{2}} \zeta(s) \varphi(s) B_{2}\left(s, s_{0}\right)\left(r_{2}(s) \eta(s)\right)^{\left(1+\frac{1}{\beta_{1} \beta_{2}}\right)}
\end{gathered}
$$

for all $\varsigma_{1}, \varsigma_{2}, T \in\left[\varsigma_{0}, \infty\right)$, where $\delta, \varphi(\varsigma)$ are defined as in Theorem 1 and $T>\varsigma_{2}>\varsigma_{1}$, then any solution of (1) is either oscillatory or satisfies $\lim _{\varsigma \rightarrow \infty} x(\varsigma)=0$.

Proof. Let (1) have a nonoscillatory solution $x(\varsigma)$ on $\left[\varsigma_{0}, \infty\right)$-say there exists $\varsigma_{1} \in\left[\varsigma_{0}, \infty\right)$ such that, for $\varsigma \geq \varsigma_{1}, x(\varsigma)>0, x(\varrho(\varsigma))>0$, and $x\left(\phi_{i}(\varsigma)\right)>0$, (3) and (4) hold, and $z(\varsigma)$ satisfies either $\left(C_{i}\right)$ or $\left(C_{i i}\right)$ and $i=1,2, \cdots, n$. Assume that $\left(C_{i}\right)$ holds. Following the same arguments as in the proof of Theorem 1, we obtain (26). Using the fact that $\varrho(\varsigma)$ is strictly increasing and $\varrho(\varsigma) \leq \phi_{i}(\varsigma)$, we have

$$
\varsigma \leq \varrho^{-1}\left(\phi_{i}(\varsigma)\right), \quad i=1,2, \cdots, n,
$$

thus, in view of the fact that $z(\varsigma)$ is increasing, we obtain

$$
\frac{z\left(\varrho^{-1}\left(\phi_{i}(\varsigma)\right)\right)}{z(\varsigma)} \geq 1, \quad i=1,2, \cdots, n
$$


Using (32) in (24), we obtain that

$$
\begin{aligned}
w^{\prime}(\varsigma) \leq & \frac{\zeta_{+}^{\prime}(\varsigma)}{\zeta(\varsigma)} w(\varsigma)+\zeta(\varsigma)\left(r_{2}(\varsigma) \eta(\varsigma)\right)^{\prime}-\zeta(\varsigma) \sum_{i=1}^{n} q_{i}(\varsigma)\left(\psi_{2}\left(\phi_{i}(\varsigma)\right)\right)^{\beta_{3}} \\
& -\beta_{3} \zeta(\varsigma) B_{2}(\varsigma, \varsigma 1)(z(\varsigma))^{\frac{\beta_{3}}{\beta_{1} \beta_{2}}-1}\left(\frac{w(\varsigma)}{\zeta(\varsigma)}-r_{2}(\varsigma) \eta(\varsigma)\right)^{1+\frac{1}{\beta_{1} \beta_{2}}}, \\
= & -\Phi_{*}(\varsigma)+C(\varsigma) \omega(\varsigma)-D(\varsigma)(w(\varsigma))^{1+\frac{1}{\beta_{1} \beta_{2}}} . \quad \text { for } \varsigma \geq \varsigma_{3} .
\end{aligned}
$$

where $C(\varsigma)$ and $D(\varsigma)$ are defined as in Theorem 1. The remainder of the proof is similar to that of Theorem 1, and so we omit it.

Theorem 4. If $\varrho(\varsigma) \leq \phi_{i}(\varsigma)$ for $i=1,2, \cdots, n$ and $\psi_{1}(\varsigma)>0, \psi_{2}(\varsigma)>0$, (5) holds. If there exists a function $\zeta \in C^{1}\left(\left[\varsigma_{0}, \infty\right), \mathbb{R}\right)$ and $\eta \in C^{1}\left(\left[\varsigma_{0}, \infty\right), \mathbb{R}\right)$ such that $r_{2} \eta \in C^{1}\left(\left[\varsigma_{0}, \infty\right), \mathbb{R}\right)$ and

$$
\limsup _{\varsigma \rightarrow \infty} \frac{1}{E\left(\varsigma, \varsigma_{*}\right)} \int_{T}^{\varsigma}\left\{E(\varsigma, s) \Phi_{*}(s)-\frac{\delta \zeta(s)|e(\varsigma, s)|^{\beta_{1} \beta_{2}+1}}{\left(E(\varsigma, s) \varphi(s) B_{2}\left(s, \varsigma_{1}\right)\right)^{\beta_{1} \beta_{2}}}\right\} d s=\infty,
$$

for all $\varsigma_{1}, \varsigma_{2}, \varsigma_{*} \in\left[\varsigma_{0}, \infty\right)$, where $\delta, \varphi(\varsigma)$ are defined as in Theorem $1, \Phi_{*}(\varsigma)$ are defined as in Theorem 2 and $\zeta_{*}>\varsigma_{2}>\varsigma_{1}$, then any solution of (1) is either oscillatory or satisfies $\lim _{\zeta \rightarrow \infty} x(\varsigma)=0$.

The following examples and comments are provided at the end of this article to illustrate the results discussed above.

Example 1. Consider the differential equation

$$
\begin{aligned}
& \left.\left(\left(\left((\varsigma-1)\left[x(\varsigma)+4 x\left(\frac{\varsigma}{2}\right)\right]^{\prime}\right)^{5}\right)^{\prime}\right)^{\frac{1}{3}}\right)^{\prime}+16^{2}(\varsigma-1)^{4} x^{3}\left(\frac{\varsigma}{2}\right)+8^{2}\left(\varsigma^{2}-3 \varsigma+2\right)^{2} x^{3}\left(\frac{\varsigma}{4}\right)=0, \quad \varsigma \geq 1 \\
& \text { where } \beta_{1}=5, \beta_{2}=1 / 3, \beta_{3}=3, r_{1}(\varsigma)=\varsigma-1, r_{2}(\varsigma)=1, p(\varsigma)=4, \varrho(\varsigma)=\varsigma / 2, \\
& q_{1}(\varsigma)=16^{2}(\varsigma-1)^{4}, q_{2}(\varsigma)=8^{2}\left(\varsigma^{2}-3 \varsigma+2\right)^{2}, \phi_{1}(\varsigma)=\varsigma / 2 \text { and } \phi_{2}(\varsigma)=\varsigma / 4 \text {. Then, we obtain } \\
& B_{1}\left(\varsigma, \varsigma_{1}\right)=B_{1}(\varsigma, 1)=\varsigma-1 ; \quad B_{2}\left(\varsigma, \varsigma_{2}\right)=B_{2}(\varsigma, 1)=1 ; \quad B_{3}\left(\varsigma, \varsigma_{2}\right)=B_{3}(\varsigma, 1)=\varsigma-1 ; \\
& B_{3}\left(\varrho^{-1}(\varsigma), \varsigma_{2}\right)=B_{3}(2 \varsigma, 1)=2 \varsigma-1 ; \quad B_{3}\left(\varrho^{-1}\left(\varrho^{-1}(\varsigma)\right), \varsigma_{2}\right)=B_{3}(4 \varsigma, 1)=4 \varsigma-1 ; \\
& B_{3}\left(\varrho^{-1}\left(\phi_{1}(\varsigma)\right), \varsigma_{2}\right)=B_{3}(2 \varsigma, 1)=2 \varsigma-1 ; \quad B_{3}\left(\varrho^{-1}\left(\phi_{2}(\varsigma)\right), \varsigma_{2}\right)=B_{3}(4 \varsigma, 1)=4 \varsigma-1 ; \\
& \text { and } \quad \begin{array}{l}
\psi_{1}(\varsigma)=\frac{1}{4}\left(1-\frac{1}{4}\right)=3 / 16>0, \\
\psi_{2}(\varsigma)=\frac{1}{4}\left(1-\frac{1}{4} \frac{4 \varsigma-1}{2 \varsigma-1}\right)=\frac{1}{16}\left(\frac{4 \varsigma-3}{2 \varsigma-1}\right) \geq \frac{1}{16}>0, \\
\Omega_{1}(\varsigma)=\sum_{i=1}^{2} q_{i}(\varsigma)\left(\psi_{1}\left(\phi_{i}(\varsigma)\right)\right)^{\beta}=16^{2}(\varsigma-1)^{4}\left(\frac{7}{64}\right)^{3}+8^{2}\left(\varsigma^{2}-3 \varsigma+2\right)^{2}\left(\frac{7}{64}\right)^{3},
\end{array}
\end{aligned}
$$

and $\delta=10.1207, \varphi(\varsigma)=m_{1}$. If we choose $\zeta(\varsigma)=\varsigma, \eta=1 / \varsigma$, it is easy to verify that all the conditions of Theorem 1 are satisfied. Hence, any solution of (35) is either oscillatory or satisfies $\lim _{\varsigma \rightarrow \infty} x(\varsigma)=0$.

Example 2. Consider the differential equation

$$
\left(\left(\left(\left((\varsigma-1)\left[x(\varsigma)+4 x\left(\frac{\varsigma}{2}\right)\right]^{\prime}\right)^{7}\right)^{\prime}\right)^{\frac{1}{5}}\right)^{\prime}+25^{2}(\varsigma-1)^{10} x^{5}\left(\frac{\varsigma}{2}\right)+25^{2}(2 \varsigma-1)^{2} x^{5}(\varsigma)=0, \quad \varsigma \geq 1
$$


where $\beta_{1}=7, \beta_{2}=1 / 5, \beta_{3}=5, r_{1}(\varsigma)=\varsigma-1, r_{2}(\varsigma)=1, p(\varsigma)=4, \varrho(\varsigma)=\varsigma / 2$, $q_{1}(\varsigma)=25^{2}(\varsigma-1)^{10}, q_{2}(\varsigma)=25^{2}(2 \varsigma-1)^{2}, \phi_{1}(\varsigma)=\varsigma / 2$ and $\phi_{2}(\varsigma)=\varsigma$. Then, we obtain

$$
\begin{aligned}
& B_{1}\left(\varsigma, \varsigma_{1}\right)=B_{1}(\varsigma, 1)=\varsigma-1 ; \quad B_{2}\left(\varsigma, \varsigma_{2}\right)=B_{2}(\varsigma, 1)=1 ; \quad B_{3}\left(\varsigma, \varsigma_{2}\right)=B_{3}(\varsigma, 1)=\varsigma-1 ; \\
& B_{3}\left(\varrho^{-1}(\varsigma), \varsigma_{2}\right)=B_{3}(2 \varsigma, 1)=2 \varsigma-1 ; \quad B_{3}\left(\varrho^{-1}\left(\varrho^{-1}(\varsigma)\right), \varsigma_{2}\right)=B_{3}(4 \varsigma, 1)=4 \varsigma-1 ;
\end{aligned}
$$

and

$$
\begin{aligned}
& \psi_{1}(\varsigma)=\frac{1}{5}\left(1-\frac{1}{5}\right)=4 / 25>0, \\
& \psi_{2}(\varsigma)=\frac{1}{5}\left(1-\frac{1}{5} \frac{4 \varsigma-1}{2 \varsigma-1}\right)=\frac{1}{25}\left(\frac{6 \varsigma-4}{2 \varsigma-1}\right) \geq \frac{1}{25}>0, \\
& \Omega_{1}(\varsigma)=\sum_{i=1}^{2} q_{i}(\varsigma)\left(\psi_{1}\left(\phi_{i}(\varsigma)\right)\right)^{\beta}=25^{2}(\varsigma-1)^{10}\left(\frac{4}{25}\right)^{3}+25^{2}(2 \varsigma-1)^{2}\left(\frac{4}{25}\right)^{3},
\end{aligned}
$$

and $\delta=\left(\frac{7}{25}\right)^{7 / 5}\left(\frac{5}{12}\right)^{12 / 5}, \varphi(\varsigma)=m_{1}$. If we choose $\zeta(\varsigma)=1, \eta=1 / \varsigma$, it is easy to verify that all the conditions of Theorem 3 are satisfied. Hence, any solution of (36) is either oscillatory or satisfies $\lim _{\varsigma \rightarrow \infty} x(\varsigma)=0$.

\section{Conclusions}

We established new oscillation theorems for (1) under the assumptions of $\varrho(\varsigma) \geq \phi_{i}(\varsigma)$ and $\varrho(\varsigma) \leq \phi_{i}(\varsigma)$ for $i=1,2, \cdots, n$ when $p(\varsigma) \geq 1$. The symmetry plays an important and fundamental role in the study of the oscillation of the solutions of equations. The main outcomes are proved via the means of generalized riccati substitution, an integral averaging condition under the assumptions of $\int_{\varsigma_{0}}^{\infty} r_{i}^{-1 / \beta_{i}}(s) d s=\infty$ for $i=1,2$. Two examples are given to prove the significance of the new theorems. Furthermore, we can try to obtain new oscillation results of $(1)$ if $z(\varsigma):=x(\varsigma)+\sum_{i=1}^{2} p_{i}(\varsigma) x\left(\varrho_{i}(\varsigma)\right)$ in future work.

Author Contributions: Conceptualization, M.S.K., O.B., K.A.-S., F.W. and K.N.; methodology, M.S.K., O.B., K.A.-S., F.W. and K.N.; investigation, M.S.K., O.B., K.A.-S., F.W. and K.N.; resources, M.S.K., O.B. and K.N.; data curation, M.S.K., O.B., K.N.; writing-original draft preparation, M.S.K., O.B. and K.N.; writing-review and editing, M.S.K., O.B. and K.N.; supervision, M.S.K., O.B., K.A.-S., F.W. and K.N.; project administration, M.S.K., O.B., K.A.-S., F.W. and K.N.; funding acquisition, M.S.K., O.B., K.A.-S., F.W. and K.N. All authors read and agreed to the published version of the manuscript.

Funding: This research received no external funding.

Institutional Review Board Statement: Not applicable.

Informed Consent Statement: Not applicable.

Acknowledgments: The authors thank the reviewers for their useful comments, which led to the improvement of the content of the paper.

Conflicts of Interest: The authors declare no conflict of interest.

\section{References}

1. Hale, J.K. Theory of Functional Differential Equations; Springer: New York, NY, USA, 1977.

2. Baculíková, B.; Dzurina, J. Oscillation of third-order neutral differential equations. Math. Comput. Model. 2010, 52, $215-226$. [CrossRef]

3. Dzurina, J.; Thandapani, E.; Tamilvanan, S. Oscillation of solutions to third-order half-linear neutral differential equations. Electron. J. Differ. Equ. 2012, 29, 1-9.

4. Li, T.; Zhang, C.; Xing, G. Oscillation of Third-Order Neutral Delay Differential Equations. Abstr. Appl. Anal. 2012, $2012,569201$. [CrossRef]

5. Chatzarakis, G.E.; Grace, S.R.; Jadlovská, I.; Li, T.; Tunc, E. Oscillation criteria for third-order Emden-Fowler differential equations with unbounded neutral coefficients. Complexity 2019, 2019, 5691758. [CrossRef]

6. Tunc, E. Oscillatory and asymptotic behavior of third-order neutral differential equations with distributed deviating arguments. Electron. J. Differ. Equ. 2017, 2017, 1-12. [CrossRef] 
7. Agarwal, R.P.; Bazighifan, O.; Ragusa, M.A. Nonlinear Neutral Delay Differential Equations of Fourth-Order: Oscillation of Solutions. Entropy 2021, 23, 129. [CrossRef] [PubMed]

8. Tian, Y.; Cai, Y.; Fu, Y.; Li, T. Oscillation and asymptotic behavior of third-order neutral differential equations with distributed deviating arguments. Adv. Differ. Equ. 2015, 2015, 267. [CrossRef]

9. Philos, C.G. Oscillation theorems for linear differential equations of second order. Arch. Math. 1989, 53, 482-492. [CrossRef]

10. Graef, J.R.; Tunc, E.; Grace, S.R. Oscillatory and asymptotic behavior of a third-order nonlinear neutral differential equation. Opusc. Math. 2017, 37, 839-852. [CrossRef]

11. Jiang, Y.; Jiang, C.; Li, T. Oscillatory behavior of third-order nonlinear neutral delay differential equations. Adv. Differ. Equ. 2016, 2016, 171. [CrossRef]

12. Bazighifan, O.; Mofarreh, F.; Nonlaopon, K. On the Qualitative Behavior of Third-Order Differential Equations with a Neutral Term. Symmetry 2021, 13, 1287. [CrossRef]

13. Li, T.; Rogovchenko, Y.V. On the asymptotic behavior of solutions to a class of third-order nonlinear neutral differential equations. Appl. Math. Lett. 2020, 105, 106293. [CrossRef]

14. Chatzarakis, G.E.; Dzurina, J.; Jadlovski, I. Oscillatory properties of third-order neutral delay differential equations with noncanonical operators. Mathematics 2019, 7, 1177. [CrossRef]

15. Kumar, M.S.; Ganesan, V. Asymptotic behavior of solutions of third-order neutral differential equations with discrete and distributed delay. AIMS Math. 2020, 5, 3851-3874. [CrossRef]

16. Kumar, M.S.; Bazighifan, O.; Almutairi, A.; Chalishajar, D.N. Philos-type oscillation results for third-order differential equation with mixed neutral terms. Mathematics 2021, 9, 1021. [CrossRef]

17. AlGhamdi, A.; Bazighifan, O.; El-Nabulsi, R.A. Important Criteria for Asymptotic Properties of Nonlinear Differential Equations. Mathematics 2021, 9, 1659. [CrossRef]

18. Kumar, M.S.; Janaki, S.; Ganesan, V. Some new oscillatory behavior of certain third-order nonlinear neutral differential equations of mixed type. Int. J. Appl. Comput. Math. 2018, 4, 78. [CrossRef]

19. Tunc, E.; Grace, S.R. Oscillatory behavior of solutions to third-order nonlinear differential equations with a superlinear neutral term. Electron. J. Differ. Equ. 2020, 2020, 1-11.

20. Soliman, A.A.; Sallam, R.A.; Elbitar, A.; Hassan, A.M. Oscillation criteria of third order nonlinear neutral differential equations. Int. J. Appl. Math. Res. 2012, 1, 268-281. [CrossRef]

21. Sun, Y.; Zhao, Y. Oscillation and asymptotic behavior of third-order nonlinear neutral delay differential equations with distributed deviating arguments. J. Appl. Anal. Comput. 2018, 8, 1796-1810. 